\title{
"Vitamin D supplementation and bone health in adults with diabetic nephropathy: the protocol for a randomized controlled trial"
}

\author{
Diana R Mager ${ }^{1,2^{*}}$, Stephanie T Jackson ${ }^{1}$, Michelle R Hoffmann ${ }^{1}$, Kailash Jindal $\left.\right|^{3,4,5}$ and Peter A Senior ${ }^{3,6}$
}

\begin{abstract}
Background: Suboptimal vitamin D status is highly prevalent in Northern communities, particularly in those patients with chronic diseases such as diabetes and chronic renal disease. Emerging literature suggests that adherence to daily vitamin $\mathrm{D}$ supplementation may be an important factor influencing vitamin $\mathrm{D}$ status and overall bone health, but compliance with therapies for bone health is a major challenge. It is unknown what level of vitamin D supplementation will ameliorate or improve suboptimal vitamin D status in patients with diabetic nephropathy or contribute to improved bone health, particularly for those living in northern climates.

Methods/Design: The study purpose was to examine two different strategies of vitamin D3 supplementation; daily dosing of 2000 IU per day verses monthly dosing of 40,000 IU per month on markers of vitamin D status, bone health and to examine whether adherence, quality of life and patient satisfaction with the supplementation strategy differs between the two vitamin D strategies in adults diagnosed with diabetic nephropathy.
\end{abstract}

Discussion: The need for RCTs assessing higher doses of vitamin $D_{3}$ supplementation at varying frequencies of administration and its impact on bone health in adults with diabetes and chronic kidney disease are needed.

Trial registration: ClinicalTrials.gov NCT01476501.

Keywords: Vitamin D supplementation, Bone health, Diabetes, Kidney disease

\section{Background}

Suboptimal vitamin D status $(25(\mathrm{OH}) \mathrm{D}<75 \mathrm{nmol} / \mathrm{L})$ is associated with the development and progression of both diabetes and chronic kidney disease (CKD) [1]. Within the general North American population 16-52\% have suboptimal vitamin D status; the prevalence of vitamin D insufficiency increases to $86 \%$ in the diabetic population and those with concurrent kidney disease are 1.78-fold more likely to be vitamin D deficient [2-4]. Patients living in northern communities are at particular risk for vitamin D insufficiency due to limited sunlight exposure, further increasing their risk for low bone mineral density (BMD) and fragility fractures [3,5-10]. Recent evidence indicates that up to $40-90 \%$ of patients with stage 3-4 CKD have insufficient/

\footnotetext{
* Correspondence: mager@ualberta.ca

'Department of Agricultural, Food and Nutritional Science, University of Alberta, Edmonton, AB, Canada

${ }^{2}$ Department of Pediatrics, University of Alberta, Edmonton, AB, Canada Full list of author information is available at the end of the article
}

deficient vitamin D status $[2,5,11,12]$. By the time CKD patients reach dialysis, approximately $75 \%$ have metabolic bone disease [13].

Unlike other nutrients, recommendations for vitamin D are not based on food sources as there are very few dietary options available (e.g. fish, liver and fortified dairy products), but rather are designed to compensate for a deficiency of sunlight [14]. This places the individual living in northern climates at particular risk for inadequate vitamin $\mathrm{D}$ status, especially in the winter months when sunlight exposure is unlikely to contribute to overall vitamin D status. Most evidence suggests that when vitamin D requirements are met, it is with a combination of dietary vitamin $\mathrm{D}$ and a supplement [15-22]. Patients with diabetic nephropathy are at increased risk for poor dietary intake of vitamin $\mathrm{D}$ due to restrictions on vitamin $\mathrm{D}$ rich foods/beverages (e.g. dairy based products) as these products also have a high carbohydrate, phosphorus and/or potassium content. It is unknown what 
level of vitamin D supplementation will ameliorate or improve suboptimal vitamin $\mathrm{D}$ status in patients with diabetic nephropathy or contribute to improved bone health, particularly for those living in northern climates $[5,7,10]$. A recent study in patients with stage 3-4 CKD demonstrated that daily oral supplementation of vitamin $\mathrm{D}_{3}(1,000 \mathrm{IU} / \mathrm{d})$ for three months resulted in a mean increase in serum $25(\mathrm{OH}) \mathrm{D}$ of $25 \mathrm{nmol} / \mathrm{L}(40 \pm 15 \mathrm{nmol} / \mathrm{L}$ pre- vs. $68 \pm$ $25 \mathrm{nmol} / \mathrm{L}$ post-supplementation); a significantly lower level of $25(\mathrm{OH}) \mathrm{D}$ than is thought to optimize bone health $(>100 \mathrm{nmol} / \mathrm{L})$ [12,23-25].

Emerging literature suggests that adherence to daily vitamin D supplementation may be an important factor influencing vitamin D status, and compliance with therapies for bone health (like other asymptomatic conditions) is a major challenge [26-28]. Chronic diseases, such as poor bone health, as well as suboptimal vitamin $\mathrm{D}$ status, have been associated with reduced quality of life (QoL) [26,29-31]. Despite the potential for improved functional ability and independence, only $50-69 \%$ of individuals prescribed osteoporosis medications (e.g. bisphosphonates, vitamin $\mathrm{D}$ and calcium) comply to them regularly (e.g. consume $80 \%$ of the time), and only 25-35\% are compliant for more than one year [29-31]. This suggests that current modes of vitamin D supplementation in adults with diabetic nephropathy, particularly low dose daily administration $(<1,000 \mathrm{IU} / \mathrm{d})$, may be ineffective at optimizing vitamin $\mathrm{D}$ status. Higher daily doses $(>1,000 \mathrm{IU} / \mathrm{d})$ or the use of high dose, less frequent modes of administration (monthly vs. daily), need to be explored to ensure improved compliance to dosing strategies and adequacy of overall vitamin D status, particularly in those populations at high risk for vitamin D insufficiency and suboptimal bone health (e.g. diabetic nephropathy).

\section{Purpose}

The first study aim was to investigate the impact of daily vs. monthly vitamin $\mathrm{D}_{3}$ supplementation on vitamin $\mathrm{D}$ status and markers of bone health in adults with diabetic nephropathy. The second study aim was to compare participant adherence and satisfaction between two different vitamin $\mathrm{D}_{3}$ supplementation strategies (daily vs. monthly), and quality of life (QoL).

\section{Objectives}

1. Examine the impact of two approaches to high dose, oral, vitamin $\mathrm{D}_{3}$ supplementation (2,000 IU/d vs. $40,000 \mathrm{IU} / \mathrm{m}$ ) for 6 months on overall vitamin D status $\left(25(\mathrm{OH}) \mathrm{D}\right.$ and $\left.1,25(\mathrm{OH})_{2} \mathrm{D}\right)$ and markers of bone turnover (bone-specific alkaline phosphatase, osteocalcin, $\mathrm{N}$-telopeptide of type 1 collagen) in adult patients with diabetic nephropathy.
2. Examine daily vs. monthly vitamin $\mathrm{D}_{3}$ supplementation strategies in regards to adherence, patient satisfaction and quality of life in adult patients with diabetic nephropathy.

\section{Hypotheses}

1. Vitamin $\mathrm{D}_{3}$ supplementation (2,000 IU/d vs. $40,000 \mathrm{IU} / \mathrm{m}$ ) for 6 months will result in significantly improved overall vitamin D status and improved markers of bone health in adult patients with diabetic nephropathy. Serum 25(OH)D will increase by a minimum of $25-50 \mathrm{nmol} / \mathrm{L}$ postsupplementation. Markers of bone resorption will decrease and markers of bone formation will increase after 6 months of vitamin $\mathrm{D}_{3}$ supplementation when compared to baseline levels.

2. Monthly dosing of vitamin $\mathrm{D}_{3}(40,000 \mathrm{IU} / \mathrm{m})$ over 6 months will result in improved patient adherence and satisfaction with vitamin $\mathrm{D}_{3}$ supplementation when compared to daily dosing of vitamin $\mathrm{D}_{3}$ $(2,000 \mathrm{IU} / \mathrm{d})$ resulting in improved overall vitamin $\mathrm{D}$ status, bone health parameters, and quality of life.

\section{Methods}

\section{Study design}

The study design was a randomized, controlled, openlabel trial comparing the effectiveness of two vitamin $\mathrm{D}_{3}$ dosing strategies (monthly vs. daily) on vitamin D status and markers of bone health in adults with diabetes and nephropathy over a 6 month period. Patients were randomized to one of the two vitamin $\mathrm{D}_{3}$ supplementation strategies in blocks of 30 using a random number generator (http://www.randomizer.org) by one graduate student (SJ): once monthly $(40,000 \mathrm{IU} / \mathrm{m} ; \mathrm{n}=60)$ or once daily $(2,000 \mathrm{IU} / \mathrm{d} ; \mathrm{n}=60)$ for six months. All members of the research team (with the exception the graduate students $(\mathrm{SJ} / \mathrm{MH})$ ) were blinded to study allocation. Both vitamin $\mathrm{D}$ supplements contain vitamin $\mathrm{D}_{3}$ (cholecalciferol) in gel capsule form; Jamieson Natural Sources ${ }^{\circ}$ Vitamin D 1,000 IU Soft gel (NPN 80017530), and EURO-Pharm International Canada Inc. ${ }^{\circ}$ EURO D 10,000 IU (DIN 02253178). The two dosing regimens were: 2 capsules of 1,000 IU vitamin D daily (total dose $=2,000 \mathrm{IU} / \mathrm{d}$ ), or 4 capsules of $10,000 \mathrm{IU}$ vitamin $\mathrm{D}$ at the end of each month (total dose $=40,000 \mathrm{IU} / \mathrm{m}$ ). The daily dose was selected based on results from findings that showed that patients with diabetes with stage 3-4 CKD in Northern Alberta where supplementation with 1000 IU/D vitamin D3 resulted in serum increases of $25(\mathrm{OH})$ vitamin D less than $25 \mathrm{mmol} / \mathrm{L}$ [5,7]. A monthly dose of 40,000 IU/m vitamin $D_{3}$ was chosen to achieve equivalent supplementation to daily dosing assuming an adherence rate of $69 \%$, and 
with the goal of obtaining a serum $25(\mathrm{OH}) \mathrm{D}$ concentration of $100 \mathrm{nmol} / \mathrm{L}$ [27,32].

This study was approved by the Human Research Ethics Board at the University of Alberta (File Number: Pro00022639), has received a "No Objection Letter" from Health Canada (Control File Number 148625), and is a registered clinical trial (NCT01476501). The study was monitored by a Drug and Safety Monitoring Board (DSMB) with annual safety reports submitted as per Health Canada protocol. Reporting of study results will be according to the CONSORT (Consolidated Standards of Reporting Trials) Guidelines [33].

\section{Participants}

Patients were recruited from Northern Alberta Renal Program (NARP) clinics at Alberta Health Services (AHS) in Edmonton, Alberta between November 2011 and December 2013. This is a multidisciplinary program (endocrinologists, nephrologists, registered nurses (RN), registered dietitians (RD), pharmacists and social workers) that provides care to over 1,500 patients with diabetic nephropathy in northern Alberta. Sixty adults with diabetes and nephropathy per vitamin $D_{3}$ supplementation group were recruited into this study ( $\mathrm{n}=120$ total). Potential participants were approached by a member of the clinical team (e.g. RD or RN) and asked if a research team member could discuss this study with them. If verbal consent was provided, then a research team member contacted the patient, explained the study to them and determined their eligibility for participation in this RCT; if eligible and agreed by the patient, informed consent was signed and the baseline study appointment was booked. Subsequent study visits (e.g. 3 month and 6 month follow-up appointments) were booked via telephone calls made approximately 2 months later to follow-up with the participants and address any questions or concerns they may have had about their supplement strategy. Patient eligibility for this RCT was determined upon information available in the medical chart at time of screening. Inclusion/exclusion criteria are as follows:

\section{Inclusion criteria}

1) Adult (18-80 years) patients diagnosed with diabetes (Type 1 and Type 2) and stage 1-4 CKD (Glomerular Filtration Rate (GFR) $15-89 \mathrm{~mL} / \mathrm{min} /$ $\left.1.73 \mathrm{~m}^{2}\right)$ [34].

\section{Exclusion criteria}

1) Patients with co-morbid conditions known to affect vitamin $\mathrm{D}$ metabolism including gastrointestinal, liver, rheumatoid or bone disorders (e.g. hyperthyroidism, untreated celiac disease, cancer,
Paget's disease, sarcoidosis, malabsorption, etc.). Individuals with severe, permanent vision impairment will be excluded as this will preclude them from reading supplement labels accurately and safely. Pregnant women will be excluded as Dual-energy X-ray Absorptiometry (DXA) scans are not recommended during pregnancy. Patients weighing $>136 \mathrm{~kg}$ will be excluded as the DXA table cannot accommodate this weight.

2) Patients on drug therapy known to interfere with vitamin D (e.g. oral glucocorticoids, cholestyramine, colestipol, mineral oil, Orlistat, digoxin).

3) Patients on other forms of active D metabolites (e.g. calcitriol, vitamin D2).

4) Patients with stage 5 CKD $\left(\right.$ GFR $\left.<15 \mathrm{~mL} / \mathrm{min} / 1.73 \mathrm{~m}^{2}\right)$, receiving dialysis or on a kidney transplant list.

5) Patients with pre-existing hypercalcemia ( $>2.75 \mathrm{mmol} / \mathrm{L})$, hyperphosphatemia $(>2.0 \mathrm{mmol} / \mathrm{L})$, severe secondary hyperparathyroidism $(\mathrm{PTH}>66$ $\mathrm{pmol} / \mathrm{L})$, and serum $25(\mathrm{OH}) \mathrm{D}>200 \mathrm{nmol} / \mathrm{L}$.

6) Patients with serum $25(\mathrm{OH}) \mathrm{D}<37.5 \mathrm{nmol} / \mathrm{L}$ at time of screening to control for correction of vitamin D deficiency [5].

7) Patients undergoing strict heavy exercise for weight control and/or those who used sunscreen lotion on a regular basis.

\section{Sample size}

This sample size $(n=120)$ was based on the ability to detect a mean difference of $25-50 \mathrm{nmol} / \mathrm{L}$ in serum 25 $(\mathrm{OH}) \mathrm{D}$ from baseline levels after 6 months of vitamin $D_{3}$ supplementation in each group $(\alpha=0.05$ and $\beta=0.8)$ with an additional $15 \%$ to account for potential subject attrition $[5,8]$. Recent evidence has shown that a mean increase of $25 \mathrm{nmol} / \mathrm{L}$ in 25(OH)D with $1,000 \mathrm{IU} /$ day supplemental vitamin $\mathrm{D}_{3}$ was insufficient to promote 25 $(\mathrm{OH}) \mathrm{D}$ in excess of $100 \mathrm{nmol} / \mathrm{L}$ - the level of $25(\mathrm{OH}) \mathrm{D}$ that has been associated with having a beneficial impact on markers of bone health $[5,7,23]$. Therefore, we chose this specific vitamin $\mathrm{D}_{3}$ dose to ensure serum 25(OH)D would increase by $25-50 \mathrm{nmol} / \mathrm{L}$.

\section{Data collection}

Assessment of vitamin D status, bone health and lifestyle factors (diet, physical activity, sunlight exposure, QoL) were performed during study visits at the Clinical Research Unit (CRU) at the University of Alberta at baseline, 3 and 6 months post study enrolment between January 2012 and June 2014. Participants were given a 3 month supply of their respective vitamin $D_{3}$ supplementation strategy at baseline and again at the 3 month study visit. They were asked to return their vitamin $\mathrm{D}_{3}$ vials/pill containers to the study investigators at the 3 and 6 month study visits. At baseline, information related to patient demographics and 
anthropometrics was collected. This included age, gender, ethnicity, medications/supplement use, insulin regimen, height, weight (Health O-Meter Professional model 597KL, Pelstar LLC, Alsip, IL, USA) and BMI. Changes in these variables between appointments were documented (e.g. medications, height, weight). Bone mineral density (BMD) was measured at baseline using Dual-energy X-ray Absorptiometry (DXA); a validated tool to assess BMD (General Electric LUNAR Prodigy, version 10.5, Madison, WI, USA). Whole body scans as well as site specific scans of the lumbar spine (L1-L4) and left total hip (including femoral neck) were conducted. The precision error, expressed as a percentage coefficient of variation $(\% \mathrm{CV})$, for the Lunar DXA located in the CRU are as follows: whole body BMD 0.7\%, lumbar spine $0.9 \%$ and total hip $1.2 \%$.

Lifestyle factors were assessed at baseline, 3 and 6 months using validated tools [35-41]. These include: 1) 3-day food record to assess vitamin $D$ and calcium intake and other dietary factors known to influence vitamin D and bone health (phosphorus, carbohydrates, protein, caffeine). Dietary intake was analyzed using the Food Processor Database (Food Processor SQL, v.10.8, ESHA Research, Salem, Oregon, USA); 2) Weight-bearing physical activity records; 3) Sunlight exposure questionnaires; 4) Health related QoL (SF-36) questionnaires (www.qualitymetric.com Liscence Number QM019185 OPTUMInsight ${ }^{\mathrm{TDM}}$ ); and 5) Adherence and acceptance surveys [34-38,42-44]. Adherence to vitamin $\mathrm{D}_{3}$ supplementation was also assessed by pill counts of returned vitamin bottles at 3 and 6 months.

\section{Laboratory investigations}

To avoid risk of hypoglycemic events due to variations in appointment availability (e.g. insulin regimen incompatibility with fasting and later appointment times), random serum/plasma samples for measurement of routine clinical blood work and study blood work were collected. However, most blood samples were collected between 10 am-2 pm; approximately 2 hours post meal consumption (of either breakfast or lunch) to minimize the potential influences of variable blood collection times on bone turnover marker expression [39].

Blood samples were collected onsite at each research visit by a trained phlebotomist using validated techniques and tubes (SST gel for serum, and lithium heparin PST gel and EDTA plasma). Once collected, blood samples were immediately held at $2-8^{\circ} \mathrm{C}$ until processing by the research team or provincial laboratory system. Patients routinely receive clinical blood work to assess their glycemic control, kidney function and overall health, including: estimated GFR (eGFR), fasting/random blood glucose (FBG/RBG), urea, creatinine, hemoglobin A1c (HbA1c), calcium, albumin, phosphorus, magnesium, 25(OH)D and PTH. These variables were collected at all 3 study visits (except PTH) along with $1,25(\mathrm{OH})_{2} \mathrm{D}$ status.
Serum PTH, bone turnover markers and fibroblast growth factor-23 (FGF-23) were collected at baseline and 6 month follow-up study visits.

Routine clinical blood work, $1,25(\mathrm{OH})_{2} \mathrm{D}$ and PTH were measured by validated, specific and sensitive methodologies used by the provincial laboratory system. Estimated GFR was calculated using a validated online equation provided by K/DOQI (Modification of Diet in Renal Disease (MDRD) study group and the Chronic Kidney Disease Epidemiology Collaboration (CKD-EPI) group; http:// www.kidney.org/professionals/kdoqi/gfr_calculator.cfm). Bone turnover markers were analyzed by the research team using standardized commercial ELISA kits. After blood collection, EDTA plasma and serum clot samples (e.g. SST gel) were kept for approximately 30-60 minutes at $2-8^{\circ} \mathrm{C}$ and then centrifuged at 2,500 RPM at $4^{\circ} \mathrm{C}$ for 10 minutes (CR4.22 centrifuge, Jouan, Winchester, VA, USA). Recovered serum and plasma were aliquoted into clean micro-tubes according to volumes required for each specific assay to be tested in duplicate. Samples were stored frozen at $-80^{\circ} \mathrm{C}$ until ELISA testing, and prepared according to the manufacturer instructions for each commercial ELISA kit: serum intact osteocalcin (OC; MicroVue Osteocalcin EIA Kit, Quidel, San Diego, CA, USA), serum bone-specific alkaline phosphatase (BAP; MicroVue BAP EIA Kit, Quidel, San Diego, CA, USA), serum N-telopeptide of type 1 collagen (NTx; Osteomark NTx Serum, Wampole Laboratories, Princeton, NJ, USA), and plasma intact fibroblast growth factor-23 (FGF-23; Human Intact FGF-23 ELISA Kit, Immunotopics Inc, San Clemente, CA, USA). The intraassay (a) and inter-assay (b) coefficient of variance (CV) for these commercial kits are as follows: OC a) 4.8$10.0 \%$, b) $4.8-9.8 \%$; BAP a) $3.9-5.8 \%$, b) $5.0-7.6 \%$; NTx a) $4.6-13.99 \%$, b) $6.9-13.99 \%$; and FGF-23 a) $2.6-4.4 \%$, b) $6.1-6.5 \%$.

\section{Outcome measurements}

Outcome measurements are illustrated in Figure 1.

\section{Primary outcome variables}

\section{Serum 25(OH)D and 1,25(OH) 2 D}

Serum $25(\mathrm{OH}) \mathrm{D}$ is considered the most reliable measure of vitamin D status as it accounts for cutaneous and dietary sources of vitamin D [40].

A mean increase in serum 25(OH)D of $25-50 \mathrm{nmol} / \mathrm{L}$ was chosen as a conservative target for $25(\mathrm{OH}) \mathrm{D}$ as $1,000 \mathrm{IU} / \mathrm{d}$ has been shown to increase serum 25(OH)D by $25 \mathrm{nmol} / \mathrm{L}$ in renal patients [5]. $1,25(\mathrm{OH})_{2} \mathrm{D}$ was measured to determine concentration of active vitamin $\mathrm{D}$ in participants and explore the relationship of active vitamin $\mathrm{D}$ with $25(\mathrm{OH}) \mathrm{D}$ levels and with bone health. Vitamin D levels were measured by validated, specific and sensitive methodologies used by the provincial 


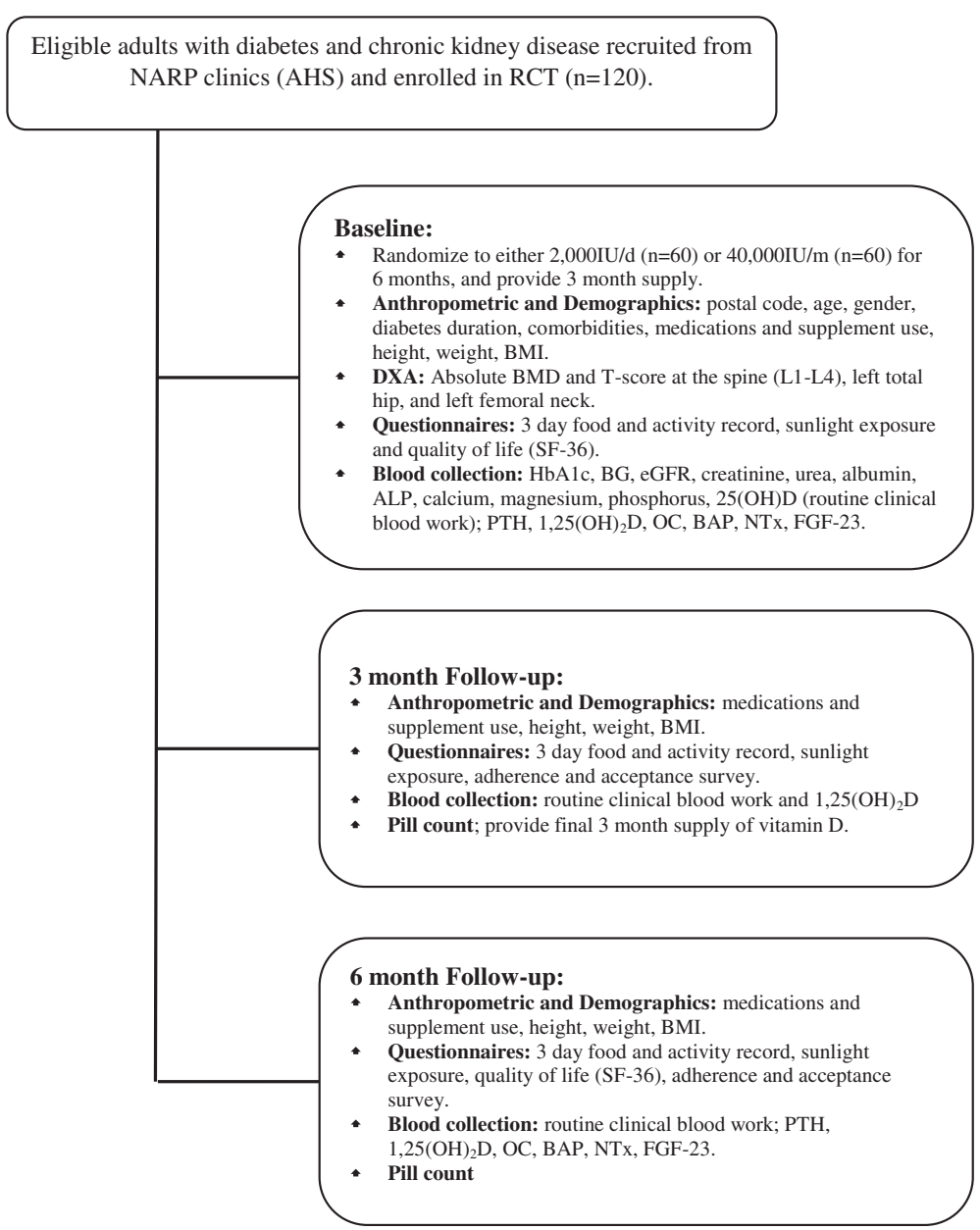

Figure 1 Study design ${ }^{1} .{ }^{1}$ Abbreviations: Northern Alberta Renal Program (NARP); Alberta Health Services (AHS); randomized controlled trial (RCT); micrograms (mcg); Body mass index (BMI); Dual X-ray absorptiometry (DXA); Bone mineral density (BMD); Hemoglobin A1c (HbA1c); random blood glucose (RBG); estimated glomerular filtration rate (eGFR); 25 -hydroxy vitamin D (25(OH)D); 1,25-dihydroxy vitamin D (1,25 $\left.(\mathrm{OH})_{2} \mathrm{D}\right)$; alkaline phosphatase (ALP); osteocalcin (OC); bone-specific alkaline phosphatase (BAP); N-telopeptide of type 1 collagen (NTx); fibroblast growth factor-23 (FGF-23).

laboratory system. The ratio of $1,25(\mathrm{OH})_{2} \mathrm{D}$ (product) to $25(\mathrm{OH}) \mathrm{D}$ (precursor) was calculated as a surrogate measure of $1 \alpha$-hydroxylase activity (product to precursor ratio).

\section{Bone health}

Bone health was assessed by measurement of BMD and plasma/serum levels of bone turnover markers. BMD was measured using DXA (non-invasive gold standard) to characterize bone health of participants at time of study entry. Bone turnover markers were measured at baseline and at 6 months; bone resorption: $\mathrm{N}$-telopeptide type $1 \mathrm{col}-$ lagen (NTx); and bone formation: bone-specific alkaline phosphatase (BAP) and osteocalcin (OC) [34]. Serum PTH and fibroblast growth factor-23 (FGF-23; measure of bone-kidney axis) were measured at baseline and 6 months to assess how serum PTH/FGF-23 concentrations change with our vitamin D supplementation strategies.

\section{Secondary outcome variables}

\section{Participant acceptance and adherence survey}

Participants completed a validated survey developed to assess adherence to drug therapy in adults with chronic disease (e.g. hypertension) that has been adapted for our specific therapy [37]. This survey consists of open and close-ended questions. This was used to assess and compare adherence to the two different dosing strategies, and consider participants' preferences and perceptions of their supplementation strategy. Adherence to the dosing regimens was also assessed by pill counts (at 3 and 6 month visits).

\section{Health-related QoL questionnaire (SF-36)}

Participants completed the SF-36 QoL questionnaire (a common and validated tool used to asses QoL in chronic disease) at baseline and 6 months after study entry [38]. This questionnaire considers parameters of 
the participants' functional capacity, social, and emotional well-being and was used to compare QoL preand-post intervention. (Liscence Number QM019185 OPTUMInsight ${ }^{\mathrm{TDM}}$ ).

\section{Dietary intake}

3-day food records (2 weekdays, 1 weekend day), a validated tool for diet analysis, were completed $[36,44]$. Responses were verified by an $\mathrm{RD}$ on the research team (blinded to study allocation) and analyzed for microand macronutrient intake (e.g. calcium, vitamin D, phosphorus, carbohydrates, protein and caffeine) using Food Processor $^{\circ}$ (Canadian Nutrient File ${ }^{\circ}$.

\section{Weight-bearing physical activity}

Analysis of the frequency/duration/effects of weightbearing physical activity utilizing a record adapted from a validated physical activity questionnaire [34,42].

\section{Sunlight exposure questionnaire/seasonal affects}

Participants completed a validated questionnaire for vitamin $\mathrm{D}$ synthesis potential according to sun exposure behaviours [43]. This questionnaire was completed to help account for seasonal variations and the impact this may have on vitamin D synthesis.

\section{Safety variables and analysis}

Safety analysis included an evaluation of the literature and the potential for the following: 1) hypercalcemia $[27,41,45] 2$ ) signs and symptoms of acute toxicity (rare) including evidence "anorexia, nausea, vomiting, fatigue, confusion, headache, weakness, renal impairment, arrhythmias, hypertension, calcification of soft tissue and hyperphosphatemia" [41].

Adverse events with vitamin $\mathrm{D}_{3}$ supplementation at these dosing levels $(40,000 \mathrm{IU} /$ month or $2000 \mathrm{IU} / \mathrm{D})$ have not been routinely reported in the literature [11,45-47]. Supplementation with active vitamin D analogues may be useful for individuals with severely diminished renal function and capacity for active hydroxylation, and is primarily used to treat severe hyperparathyroidism. However, providing already active vitamin D results in bypassing the homeostatic controls that are in place to prevent vitamin $\mathrm{D}$ toxicity (e.g. reducing 25 -hydroxylation and increasing catabolism and excretion of $25(\mathrm{OH}) \mathrm{D}$ via bile), which in turn can increase risk for vitamin D toxicity $[23,46]$. Serum phosphate $(>2.0 \mathrm{mmol} / \mathrm{L})$, calciumphosphorous product $\left(\mathrm{CaP} ;>4.4 \mathrm{mmol}^{2} / \mathrm{L}^{2}\right)$ and magnesium $(>1.0 \mathrm{mmol} / \mathrm{L})$ are all monitored as part of routine clinical care for variation from healthy ranges [41].

A data safety monitoring board (DSMB) was included within the study design. All adverse events (AE) were documented. AEs were identified as related to the study protocol (e.g. anything directly related to our vitamin D supplementation intervention, blood collection or other examinations in the study protocol) or not related to the study protocol. Serious adverse events (SAEs) were defined as significant health/safety issues (e.g. acute renal failure, death). The responsible physician (PS), University HREB, and DSMB were notified of all AE/SAE. No SAEs due to study protocol occurred in this study.

\section{Concomitant medication}

Patients were asked to continue taking their normal medications as advised by their physician, e.g. insulin, oral hypoglycaemic agents, anti-hypertensives, statins, diuretics, phosphate binders, potassium lowering agents, anemia treatment, Replavite, and/or proton-pump inhibitors. Concomitant medications were reviewed at each visit to ensure excluded medications were not prescribed in the interim. Any patients medically requiring a concomitant medication that was contraindicated were released from the study. Participants were asked to discontinue all vitamin/mineral supplements containing calcium and/or vitamin D unless prescribed for therapeutic treatment (such as calcium carbonate-containing antacids for treatment of hyperphosphatemia). Those on active vitamin $\mathrm{D}$ treatment (with other vitamin D metabolites) were excluded from the study.

\section{Rescue medication \& risk management}

Serum phosphate $(>2.0 \mathrm{mmol} / \mathrm{L}), \mathrm{CaP}$ product $\left(>4.4 \mathrm{mmol}^{2} /\right.$ $\left.\mathrm{L}^{2}\right)$ and magnesium $(>1.0 \mathrm{mmol} / \mathrm{L})$ were monitored throughout the study [48]. Vitamin D toxicity was addressed in the following ways: a) discontinuation of vitamin $\mathrm{D}_{3}$ supplement and b) notification to the Qualified Investigator (PS) and other members of the health care team in NARP/DNPC.

The following were standard responses to vitamin D toxicity in our population: 1) Serum and urine electrolytes, renal function, electrocardiogram, and fluid balance monitoring and maintenance [38]. 2) If necessary, additional measures may have been taken to enhance excretion/metabolism of the vitamin D including: IV administration of furosemide; corticosteroid, bisphosphonate or calcitonin therapy; hemo- or peritoneal dialysis [43]. If hypersensitivity to vitamin $\mathrm{D}_{3}$ occurred which included anaphylaxis, then appropriate treatment with epinephrine and ventilation would have been provided as needed.

Adverse events were reported immediately to the responsible physician (PS) or the on-call endocrinologist/ nephrologist was notified as per standard clinical care. Participants were taken to the University of Alberta Hospital, AHS if requiring immediate medical treatment.

\section{Premature withdrawal}

Participants were able to voluntarily withdraw from the trial at any time without any negative consequences to 
their clinical care. Any and all reasons for participant drop out were documented. Any individual demonstrating clinical signs and symptoms of vitamin D toxicity and/or an SAE related to the study protocol resulted in: 1) vitamin $D_{3}$ supplementation discontinued, 2) responsible physician (PS) notified so the patient received treatment for toxicity (as above), 3) notification of the DSMB and Health Canada, and 4) patient participation in the study was discontinued. New medical diagnosis arising precluding the ability to continue in the study was an additional reason for premature withdrawal. This was assessed by the qualified investigator (PS).

\section{Statistical analysis}

Data analysis was performed on an intention-to treat basis as well as a per-protocol basis. Analyses were performed using Microsoft Excel 2010 and Statistical Analysis Software (SAS; version 9.3 SAS Institute, Cary, NC, USA). Statistical significance was determined at $\mathrm{p}<0.05$. Continuous variables were expressed as mean, median, ranges, and standard error (SE) or standard deviation (SD). The differences between dosing type (daily vs. monthly) over the intervention period were assessed by repeated measures analysis of variance, followed by a post-hoc pair-wise t-test with Bonferroni corrections to assess for within and between group comparisons. Potential risk factors for the development of vitamin $\mathrm{D}$ insufficiency (serum 25(OH)D $<75 \mathrm{nmol} / \mathrm{L}$ ) and poor bone health (T-scores $<-2 \mathrm{SD}$ ) in adults with diabetic nephropathy include bone turnover, vitamin D intake, vitamin D status (serum levels of 25(OH)D and 1,25 $\left.(\mathrm{OH})_{2} \mathrm{D}\right)$, age, gender, ethnicity, severity of kidney disease, diabetic management/control, exposure to sunlight, level of activity, and psychosocial and lifestyle factors.

Continuous and categorical variables (e.g. 25(OH)D, lab parameters, BMD T-scores, adherence scores) were quantitatively analyzed, and open-ended questions regarding participants' perceived facilitators and barriers were categorized into key themes. Bivariate and univariate analysis were done to assess the potential effect of these variables on vitamin $\mathrm{D}$ status and bone health (bone turnover markers).Variables shown to be associated with a poor vitamin $\mathrm{D}$ status were assessed using multivariate logistic regression models to determine risk for development of vitamin D deficiency and poor bone health. Regression analysis was also conducted to assess correlation between biochemical parameters (PTH, 25 $(\mathrm{OH}) \mathrm{D}, 1,25(\mathrm{OH})_{2} \mathrm{D}$, calcium, phosphorus, magnesium, albumin, eGFR, RBG) and bone turnover markers. Analysis of variance was performed to assess for significant differences in vitamin $\mathrm{D}$ status and bone turnover markers over the intervention period in both groups. Regression analysis was performed with serum 25(OH)D and plasma PTH as categorical variables; e.g. 25(OH)D sorted by greater/less than $75 \mathrm{nmol} / \mathrm{L}$ and plasma $\mathrm{PTH}$ sorted by greater/less than $7.15 \mathrm{pmol} / \mathrm{L}$, the optimal concentrations for bone health $[5,26]$. Where necessary, vitamin D was adjusted for potential confounding variables (e.g. age, gender, ethnicity, disease severity) using an analysis of co-variance. When adherence to daily vitamin D dosing regimen was greater or less than $69 \%$, the variation in compliance was also accounted for in an analysis of co-variance. For variables demonstrating skewed distributions a logarithmic transformation was used to normalize the data.

\section{Data management and validation}

Prior to data entry, these individuals underwent ethics training and evaluation related to protecting participant information and health, including online University of Alberta and National Institute of Health ethics training and testing, criminal record checks, and ensuring up-todate immunizations. Source data was coded and kept in a locked filing cabinet within the Clinical Research Unit, University of Alberta. Electronic files were encrypted and kept in a password protected computer according to University of Alberta (Faculty of Medicine) encryption policy [49].

The electronic data was audited in a timely manner to ensure any discrepancies were addressed and the potential for future discrepancies was reduced. A variety of standard operating procedures (SOP) were developed to support data analysis. Discrepant results were compared with source records, and amendments were made to the electronic records as necessary. All data entry was cross verified by one trained volunteer/graduate student, along with the primary graduate students involved in the project.

\section{Discussion}

Vitamin D is a nutrient of concern for individuals with diabetes and nephropathy, particularly for those living in northern climates with limited sunlight exposure. Dietary restriction of vitamin D-rich food sources (e.g. dairy products) is a common issue due to concerns around phosphorus, potassium and carbohydrate content. Avoidance of sunlight is also common due to instructions for concomitant medications. Vitamin D supplementation is required in this population, yet the most effective dose and most efficient dosing strategy is unknown. Low adherence rates to routine vitamin D supplementation is also an inherent challenge and therefore developing alternative approaches such as alterations in dosing frequency (monthly verses daily) is also an important consideration [26]. Previous studies have been limited by small sample size, short intervention duration or retrospective design. This study prospectively investigated the impact of two different oral vitamin $\mathrm{D}_{3}$ supplementation dosing strategies $(2,000 \mathrm{IU} / \mathrm{d}$ vs. 
$40,000 \mathrm{IU} / \mathrm{m}$ ) for 6 months on markers of vitamin D status and bone health in a large group of adults with diabetes and nephropathy.

Measurement of markers of dynamic expression of bone turnover, enables evaluation of changes over time versus assessment of static changes in bone physiology that could be observed via DXA [50] is a conferred added strength. Markers of collagenous bone resorption (e.g. serum NTx), bone formation (e.g. serum BAP and $\mathrm{OC}$ ), and the bone-kidney axis/mineral homeostasis (e.g. plasma FGF-23) will allow for exploration of dynamic bone remodeling during vitamin $\mathrm{D}$ supplementation. These markers have also been suggested as valuable tools for assessing adherence to bone health therapies [51].

Conferred additional strengths to the study design include the evaluation of socio-demographic, co-morbidities, concomitant medication use and quality of life factors; all factors known to influence adherence to vitamin supplementation. Ongoing analysis will enable a comprehensive evaluation of both lifestyle and physiological factors that influence response to vitamin D supplementation in this population. Dietary intake will be analyzed using validated methodologies for its contribution to total vitamin D intake and status, as well as intake of other nutrients that are known to impact bone health (e.g. calcium, magnesium, phosphorus). Finally, participants were enrolled throughout two calendar years, thus accounting for seasonal variations in vitamin D status. They completed sunlight exposure and weight-bearing physical activity questionnaires at each appointment so that potential impact of sunlight exposure during the different seasons as well as indoor and outdoor activity can be assessed for impact on vitamin D status and BMD.

Increasing evidence suggests that current recommendations for vitamin D intake are inadequate, particularly for populations at risk for suboptimal vitamin D status, such as those with diabetes, kidney disease or living in northern climates [3,5,7,27]. Moreover, the need for RCTs assessing higher doses of vitamin $\mathrm{D}_{3}$ supplementation at varying frequencies of administration and its impact on bone health in this population is supported by the research community $[3,12,27,35,52,53]$. The present study will help fill this gap in the literature, and elucidate an appropriate vitamin $\mathrm{D}_{3}$ supplementation dose for improved vitamin $\mathrm{D}$ status and bone health in adults with diabetes and nephropathy $[12,27,35,52,53]$.

\footnotetext{
Abbreviations

AE: Adverse event; ALP: Alkaline phosphatase; AHS: Alberta health services; BAP: Bone-specific Alkaline Phosphatase; BMD: Bone mineral density; BMI: Body mass index; CONSORT: Consolidated standards of reporting trials; CKD: Chronic kidney disease; CRU: Clinical research unit; d: Daily group; DNPC: Diabetic nephropathy prevention clinic; DSMB: Data safety monitoring board; DXA: Dual energy x-ray absorptiometry; ESRD: End-stage renal disease; FBG: Fasting blood glucose; FGF-23: Fibroblast growth factor - 23; GFR: Glomerular filtration rate; HbA1c: Hemoglobin A1c; HREB: Human
}

research ethics board; K/DOQI: Kidney disease outcomes quality initiative; m: Monthly group; NARP: Northern Alberta renal program; NTx: Ntelopeptide of type 1 collagen; OC: Osteocalcin; PTH: Parathyroid hormone; QoL: Quality of life; RBG: Random blood glucose; RCT: Randomized controlled trial; RD: Registered dietitian; RIC: Renal insufficiency clinic; RN: Registered nurse; SAE: Serious adverse event; SAS: Statistical analysis software; SF-36: 36-item short form questionnaire; 1,25(OH) $2 \mathrm{D}: 1,25$ dihydroxyvitamin D; calcitriol; 25(OH)D: 25-hydroxyvitamin D; calcidiol.

\section{Competing interests}

None to report. Vitamin D suppliers (Jamieson Natural Sources ${ }^{\circledast} /$ EURO-Pharm International Canada Inc. ${ }^{\oplus}$ ) had no involvement in study design/implementation, statistical analyses or manuscript preparation.

\section{Authors' contributions}

DM: Study design, grant, ethics and Health Canada submissions, supervised data collection (SJ, MH), data analysis, co-wrote manuscript, reviewed all version of the paper. SJ: Study design, grant, ethics and Health Canada Submissions, data collection, co-wrote manuscript. $\mathrm{MH}$ : Data collection, reviewed and contributed to manuscript. KJ: Contributed to study design, subject recruitment, manuscript revisions and submission. PS: Contributed to study design, reviewed grant, ethics and Health Canada submissions, assisted with subject recruitment, data collection and reviewed and contributed to manuscript submissions. All authors read and approved the final manuscript.

\section{Acknowledgments}

Funding sources for this RCT included Canadian Kidney Foundation Allied Health Operating Grant (KFOC120024) and Food and Health Innovation Funding from the University of Alberta. Scholarship funding by the $\mathrm{G}$. Woodrow Wirtanen Studentship, Alberta Diabetes Institute (SJ), Anthony Fellowship in Clinical Nutrition (SJ), Walter H Johns Graduate Fellowship (SJ) at the University of Alberta and the Canadian Institute of Health Research (SJ) is gratefully acknowledged. The authors wish to acknowledge the in-kind contribution of the Vitamin D supplements from Jamieson Natural Products ${ }^{\circledR}$ (1000 IU/D get capsules) and Euro Pharm International ${ }^{\oplus}$ (10,000 IU gel capsules). Special thanks to Abeer Alzaben MSc PhD (candidate) for her assistance with dietary analysis, Ping Li RD for assistance with subject recruitment and the patients and families for participating in this study.

\section{Author details}

${ }^{1}$ Department of Agricultural, Food and Nutritional Science, University of Alberta, Edmonton, $\mathrm{AB}$, Canada. ${ }^{2}$ Department of Pediatrics, University of Alberta, Edmonton, AB, Canada. ${ }^{3}$ Diabetic Nephropathy Prevention Clinic, Alberta Health Services, Edmonton, AB, Canada. ${ }^{4}$ Northern Alberta Renal Program, Edmonton, $A B$, Canada. ${ }^{5}$ Department of Nephrology, University of Alberta, Edmonton, $A B$, Canada. ${ }^{6}$ Department of Endocrinology, University of Alberta, Edmonton, $\mathrm{AB}$, Canada.

Received: 28 May 2014 Accepted: 6 August 2014

Published: 12 August 2014

\section{References}

1. Pittas AG, Lau J, Hu FB, Dawson-Hughes B: The role of vitamin D and calcium in type 2 diabetes. A systematic review and meta-analysis. J Clin Endocrinol Metab 2007, 92(6):2017-2029.

2. Diaz VA, Mainous AG 3rd, Carek PJ, Wessell AM, Everett CJ: The association of vitamin $D$ deficiency and insufficiency with diabetic nephropathy: implications for health disparities. J Am Board Fam Med 2009, 22(5):521-527.

3. Genuis SJ, Schwalfenberg GK, Hiltz MN, Vaselenak SA: Vitamin D status of clinical practice populations at higher latitudes: analysis and applications. Int J Environ Res Public Health 2009, 6(1):151-173.

4. Roth DE, Martz $P$, Yeo R, Prosser C, Bell M, Jones AB: Are national vitamin D guidelines sufficient to maintain adequate blood levels in children? Can J Public Health 2005, 96(6):443-449.

5. Rucker D, Tonelli M, Coles MG, Yoo S, Young K, McMahon AW: Vitamin D insufficiency and treatment with oral vitamin D3 in northern-dwelling patients with chronic kidney disease. J Nephrol 2009, 22(1):75-82.

6. Hadjidakis DJ, Androulakis II: Bone remodeling. Ann N Y Acad Sci 2006, 1092:385-396.

7. Rucker D, Allan JA, Fick GH, Hanley DA: Vitamin D insufficiency in a population of healthy western Canadians. CMAJ 2002, 166(12):1517-1524. 
8. Hadjidakis DJ, Raptis AE, Sfakianakis M, Mylonakis A, Raptis SA: Bone mineral density of both genders in Type 1 diabetes according to bone composition. J Diabetes Complications 2006, 20(5):302-307.

9. Miazgowski T, Czekalski S: A 2-year follow-up study on bone mineral density and markers of bone turnover in patients with long-standing insulin-dependent diabetes mellitus. Osteoporos Int 1998, 8(5):399-403.

10. Holick MF: Optimal vitamin D status for the prevention and treatment of osteoporosis. Drugs Aging 2007, 24(12):1017-1029.

11. Gal-Moscovici A, Sprague SM: Use of vitamin D in chronic kidney disease patients. Kidney Int 2010, 78(2):146-151.

12. Stavroulopoulos A, Porter CJ, Roe SD, Hosking DJ, Cassidy MJ: Relationship between vitamin $\mathrm{D}$ status, parathyroid hormone levels and bone mineral density in patients with chronic kidney disease stages 3 and 4. Nephrology (Carlton) 2008, 13(1):63-67.

13. Bailie GR, Massry SG: Clinical practice guidelines for bone metabolism and disease in chronic kidney disease: an overview. Pharmacotherapy 2005, 25(12):1687-1707.

14. Vieth R: Why the optimal requirement for Vitamin D3 is probably much higher than what is officially recommended for adults. J Steroid Biochem Mol Biol 2004, 89-90(1-5):575-579.

15. Whiting SJ, Langlois KA, Vatanparast $H$, Greene-Finestone LS: The vitamin D status of Canadians relative to the 2011 dietary reference intakes: an examination in children and adults with and without supplement use. Am J Clin Nutr 2011, 94(1):128-135.

16. Vatanparast H, Calvo MS, Green TJ, Whiting SJ: Despite mandatory fortification of staple foods, vitamin D intakes of Canadian children and adults are inadequate. J Steroid Biochem Mol Biol 2010, 121(1-2):301-303.

17. Poliquin S, Joseph L, Gray-Donald K: Calcium and vitamin D intakes in an adult Canadian population. Can J Diet Pract Res 2009, 70(1):21-27.

18. Barake $\mathrm{R}$, Weiler $\mathrm{H}$, Payette $\mathrm{H}$, Gray-Donald K: Vitamin D supplement consumption is required to achieve a minimal target 25 -hydroxyvitamin D concentration of $>$ or $=75 \mathrm{nmol} / \mathrm{L}$ in older people. J Nutr 2010, 140(3):551-556.

19. Gozdzik A, Barta JL, Wu H, Wagner D, Cole DE, Vieth R, Whiting S, Parra EJ: Low wintertime vitamin $D$ levels in a sample of healthy young adults of diverse ancestry living in the Toronto area: associations with vitamin D intake and skin pigmentation. BMC Public Health [electronic resource] 2008, 8:336.

20. Mark S, Lambert M, Delvin EE, O'Loughlin J, Tremblay A, Gray-Donald K: Higher vitamin D intake is needed to achieve serum 25(OH)D levels greater than $50 \mathrm{nmol} / \mathrm{l}$ in Quebec youth at high risk of obesity. Eur J Clin Nutr 2011, 65(4):486-492.

21. Anderson LN, Cotterchio M, Boucher BA, Knight JA, Block T: Vitamin D intake from food and supplements among Ontario women based on the US block food frequency questionnaire with and without modification for Canadian food values. Can J Public Health 2010, 101(4):318-321.

22. Weiler HA, Lowe J, Krahn J, Leslie WD: Osteocalcin and vitamin D status are inversely associated with homeostatic model assessment of insulin resistance in Canadian Aboriginal and white women: the First Nations Bone Health Study. J Nutr Biochem 2013, 24(2):412-418.

23. Vieth R: Vitamin D, supplementation, 25-hydroxyvitamin D concentrations, and safety. Am J Clin Nutr 1999, 69(5):842-856.

24. Weaver CM, Fleet JC: Vitamin D requirements: current and future. Am J Clin Nutr 2004, 80(6 Suppl):1735S-1739S.

25. Gomez-Alonso C, Naves-Diaz ML, Fernandez-Martin JL, Diaz-Lopez JB, Fernandez-Coto MT, Cannata-Andia JB: Vitamin D status and secondary hyperparathyroidism: the importance of 25-hydroxyvitamin D cut-off levels. Kidney Int Suppl 2003, 85:S44-S48.

26. Iversen MD, Vora RR, Servi A, Solomon DH: Factors affecting adherence to osteoporosis medications: a focus group approach examining viewpoints of patients and providers. J Geriatr Phys Ther 2011, 34(2):72-81.

27. Bischoff-Ferrari HA: How to select the doses of vitamin D in the management of osteoporosis. Osteoporos Int 2007, 18(4):401-407.

28. Sanfelix-Genoves J, Gil-Guillen VF, Orozco-Beltran D, Giner-Ruiz V, PertusaMartinez S, Reig-Moya B, Carratalá C: Determinant factors of osteoporosis patients' reported therapeutic adherence to calcium and/or vitamin $D$ supplements: a cross-sectional, observational study of postmenopausal women. Drugs Aging 2009, 26(10):861-869.

29. Jindal K, MacNair L, Senior P: A collaborative approach to diabetes nephropathy prevention. Alta RN 2005, 61(9):10-11.

30. Ulitsky A, Ananthakrishnan AN, Naik A, Skaros S, Zadvornova Y, Binion DG, Issa M: Vitamin D deficiency in patients with inflammatory bowel disease: association with disease activity and quality of life. Jpen 2011, 35(3):308-316

31. Nicholas DB, Picone G, Selkirk EK: The lived experiences of children and adolescents with end-stage renal disease. Qual Health Res 2011, 21(2):162-173.

32. Mehrotra R, Kermah D, Budoff M, Salusky IB, Mao SS, Gao YL, Takasu J, Adler S, Norris K: Hypovitaminosis D in chronic kidney disease. Clin J Am Soc Nephrol 2008, 3(4):1144-1151.

33. Moher D, Hopewell S, Schulz KF, Montori V, Gotzsche PC, Devereaux PJ, Elbourne D, Egger M, Altman DG, CONSORT: CONSORT 2010 explanation and elaboration: updated guidelines for reporting parallel group randomised trials. J Clin Epidemiol 2010, 63(8):e1-e37.

34. Crocker PR, Bailey DA, Faulkner RA, Kowalski KC, McGrath R: Measuring general levels of physical activity: preliminary evidence for the physical activity questionnaire for older children. Med Sci Sports Exerc 1997, 29 (10):1344-1349.

35. Levin A, Hemmelgarn B, Culleton B, Tobe S, McFarlane P, Ruzicka M, Burns K, Manns B, White C, Madore F, Moist L, Klarenbach S, Barrett B, Foley R, Jindal K, Senior P, Pannu N, Shurraw S, Akbaria A, Cohn A, Reslerova M, Deved V, Mendelssohn D, Nesrallah G, Kappel J, Tonelli M, Canadian Sciety of Nephrology: Guidelines for the management of chronic kidney disease. CMAJ 2008, 179(11):1154-1162.

36. Day N, McKeown N, Wong M, Welch A, Bingham S: Epidemiological assessment of diet: a comparison of a 7-day diary with a food frequency questionnaire using urinary markers of nitrogen, potassium and sodium. Int J Epidemiol 2001, 30(2):309-317.

37. Kim MT, Hill MN, Bone LR, Levine DM: Development and testing of the hill-bone compliance to high blood pressure therapy scale. Prog Cardiovasc Nurs 2000, 15(3):90-96.

38. Wang W, Tonelli M, Hemmelgarn B, Gao S, Johnson JA, Taub K, Manns B, Alberta Kidney Disease Network: The effect of increasing dialysis dose in overweight hemodialysis patients on quality of life: a 6-week randomized crossover trial. Am J Kidney Dis 2008, 51(5):796-803.

39. Brown JP, Albert C, Nassar BA, Adachi JD, Cole D, Davison KS, Dooley KC, Don-Wauchope A, Douville P, Hanley DA, Jamal SA, Josse R, Kaiser S, Krahn J, Krause R, Kremer R, Lepage R, Letendre E, Morin S, Ooi DS, Papaioaonnou A, Ste-Marie LG: Bone turnover markers in the management of postmenopausal osteoporosis. Clin Biochem 2009, 42(10-11):929-942.

40. Vieth $\mathrm{R}$ : What is the optimal vitamin D status for health? Prog Biophys Mol Biol 2006, 92(1):26-32.

41. Canadian Pharmacists Association: Compendium of Pharmaceuticals and Specialties: The Canadian Drug Reference for Health Professionals Version 46. Canadian Pharmacists Association; 2010.

42. FitzGerald $L$, Carpenter $C$ : Bone mineral density results influencing healthrelated behaviors in male athletes at risk for osteoporosis. $J$ Clin Densitom 2010, 13(3):256-262.

43. Termorshuizen F, Wijga A, Garssen J, Den Outer PN, Slaper H, Van Loveren $\mathrm{H}$ : Exposure to solar ultraviolet radiation in young Dutch children: assessment by means of a 6-week retrospective questionnaire. $J$ Expo Anal Environ Epidemiol 2002, 12(3):204-213.

44. Freedman LS, Midthune D, Carroll RJ, Krebs-Smith S, Subar AF, Troiano RP, Dodd K, Schatzkin A, Bingham SA, Ferrari P, Kipnis V: Adjustments to improve the estimation of usual dietary intake distributions in the population. J Nutr 2004, 134(7):1836-1843.

45. Wu F, Staykova T, Horne A, Clearwater J, Ames R, Mason B, Orr-Walker B, Gamble G, Scott M, Reid I: Efficacy of an oral, 10-day course of high-dose calciferol in correcting vitamin D deficiency. N Z Med J 2003, 116(1179):U536.

46. Houghton LA, Vieth R: The case against ergocalciferol (vitamin D2) as a vitamin supplement. Am J Clin Nutr 2006, 84(4):694-697.

47. Vieth R, Fraser D: Vitamin D insufficiency: no recommended dietary allowance exists for this nutrient. CMAJ 2002, 166(12):1541-1542.

48. Vieth $\mathrm{R}$ : Critique of the considerations for establishing the tolerable upper intake level for vitamin D: critical need for revision upwards. J Nutr 2006, 136(4):1117-1122.

49. Faculty of Medicine and Dentistry UoA: Encryption Policy; 2011. Available from: http://www.industrymailout.com/Industry/Home/5882/26546/images/ Encryption_Policy_Nov_1_2011.pdf?utm_source=mailoutinteractive\&utm_ medium=email\&utm_campaign=Mandatory+self-declaration+for+data+ protection.

50. Post TM, Cremers SC, Kerbusch T, Danhof M: Bone physiology, disease and treatment: towards disease system analysis in osteoporosis. Clin Pharmacokinet 2010, 49(2):89-118. 
51. Civitelli R, Armamento-Villareal R, Napoli N: Bone turnover markers: understanding their value in clinical trials and clinical practice. Osteoporos Int 2009, 20(6):843-851.

52. Kim MJ, Frankel AH, Donaldson M, Darch SJ, Pusey CD, Hill PD, Mayr M, Tam FW: Oral cholecalciferol decreases albuminuria and urinary TGF-beta1 in patients with type 2 diabetic nephropathy on established renin-angiotensin-aldosterone system inhibition. Kidney Int 2011, 80(8):851-860.

53. Zisman AL, Wolf M: Recent advances in the rapidly evolving field of fibroblast growth factor 23 in chronic kidney disease. Curr Opin Nephrol Hypertens 2010, 19(4):335-342.

doi:10.1186/1472-6823-14-66

Cite this article as: Mager et al:: "Vitamin D supplementation and bone health in adults with diabetic nephropathy: the protocol for a randomized controlled trial". BMC Endocrine Disorders 2014 14:66.

\section{Submit your next manuscript to BioMed Central and take full advantage of:}

- Convenient online submission

- Thorough peer review

- No space constraints or color figure charges

- Immediate publication on acceptance

- Inclusion in PubMed, CAS, Scopus and Google Scholar

- Research which is freely available for redistribution 